\title{
Populações Celulares Periféricas (CD4+, CD8+ e CD19+) em Indivíduos Dislipidémicos
}

\author{
CD4+, CD8+ AND CD19+ Cells at Individuals with Dyslipidemia
}

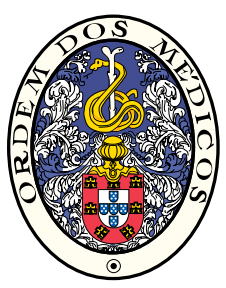

\author{
José PEREIRA de MOURA 1 , Manuel SANTOS ROSA², Vera ALVES ${ }^{2}$, Anabela MOTA PINTO ${ }^{3}$, Victor RODRIGUES ${ }^{4}$, \\ José Manuel SILVA ${ }^{1}$, J.J. ALVES de MOURA ${ }^{1}$ \\ Acta Med Port 2013 Nov-Dec;26(6):676-682
}

\section{RESUMO}

Introdução: Os mecanismos imunológicos e inflamatórios têm um papel crucial no desenvolvimento da aterosclerose e na sua tradução clínica. São inúmeros os estudos que procuraram relacionar os mais diversos marcadores inflamatórios - leucócitos, proteína C reactiva, interleucinas, quimiocinas, moléculas de adesão, metaloproteinases, etc - com os factores de risco clássicos da aterosclerose, a formação da placa e os fenómenos clínicos. Não são tantos, que tenhamos conhecimento, os trabalhos que analisaram o comportamento das diversas células mononucleares na fisiopatologia da aterosclerose. Sendo os monócitos/macrófagos e os linfócitos células fundamentais no desencadear e posterior evolução desta doença vascular, procurámos determinar as percentagens das diversas populações celulares periféricas em indivíduos dislipidémicos e em normolipidémicos.

Material e Métodos: Por citometria de fluxo, determinámos em indivíduos com dislipidemia e num grupo controlo, as concentrações no sangue periférico dos CD3+, CD4+, CD8+, CD19+, CD56+, CD56CD8+, DN, CD25+, CD26+, CD25CD3+, CD26CD3+, CD25CD26CD3+, CCR5+, CCR5CD3+, CCR5CD4+, HLADR+, HLADRCD4+, HLADRCD8h+, HLADRCD8low+, HLADRCD8+, CD95+, CD95CD95L+, CD3CD95+, CD3CD95L+, CD62L+, CD3CD62L+, CD69+, CD69CD3+ e CD69CD4+.

Resultados: Embora na sua grande maioria não tenham sido encontradas diferenças significativas entre os dois grupos de participantes, verificaram-se em algumas populações celulares, resultados que nos mereceram alguns comentários. Neste artigo debruçámo-nos apenas sobre as populações positivas para os CD4, CD8 e CD19.

Discussão: A menor concentração das células CD4+ na nossa população de dislipidémicos foi aparentemente inesperada devido ao relacionamento existente entre este tipo celular, os factores de risco e a aterosclerose. Não foram determinados os subtipos Th1 e Th2, nem a população de células reguladoras CD4+CD25+, que poderiam explicar a menor percentagem de células CD4+ nos nossos dislipidémicos. Relativamente às células CD8+ e CD19+, apresentaram percentagens sobreponíveis nos dois grupos de participantes. Conclusões: Apesar das limitações evidenciadas em resultado da reduzida dimensão das populações, foi possível demonstrar uma redução em algumas moléculas após aplicação de ácido acetilsalicílico.

Palavras-chave: Células Sanguíneas; Citometria de Fluxo; Dislipidemia; Marcadores Biológicos.

\section{ABSTRACT}

Introduction: The past decade has witnessed an increasing recognition that inflammatory mechanisms play a central role in the pathogenesis of atherosclerosis and its complications. Recently, attention was focused on the potential role of plasma markers of inflammation as risk predictors among those at risk for cardiovascular events. Of these potential markers, C-reactive protein (CRP), IL6, metalloproteinases, ICAM, VCAM and other molecules, have been extensively studied. On the other hand, to our knowledge, there are only a few studies on the role of inflammatory cells, like $T$ and B lymphocytes in the atherosclerosis.

Material and Methods: By Flow Cytrometry analysis we have determined on dyslipidemic people and on a control group, the percentage of some peripheral inflammatory cells, like CD3+, CD4+, CD8+, CD19+, CD56+, CD56CD8+, DN, CD25+, CD26+, CD25CD3+, CD26CD3+, CD25CD26CD3+, CCR5+, CCR5CD3+, CCR5CD4+, HLADR+, HLADRCD4+, HLADRCD8h+, HLADRCD8low+, HLADRCD8+, CD95+, CD95CD95L+, CD3CD95+, CD3CD95L+, CD62L+, CD3CD62L+, CD69+, CD69CD3+ e CD69CD4+.

Results: In the present study we have particularly studied the percentage of CD4+, CD8+ and CD19+ cells. The CD4+ cells have been significantly reduced in the people with dyslipidemia.

Discussion: We do not know the peripheral numbers of the subtype Th1 and Th2, neither the percentage of CD4+CD25+ cells (regulatory T cells). We have not find any differences on the percentage from the CD8+ and CD19+ cells.

Conclusions: In spite of the identified limitations resulting from the small-sized samples, it was possible to show a reduction of some molecules after application of acetylsalicylic acid.

Keywords: Biological Markers; Blood Cells; Dyslipidemias; Flow Cytometry.

\section{INTRODUÇÃO}

A aterosclerose, envolvendo as artérias de médio e grande calibre, inicialmente interpretada como uma doença essencialmente degenerativa, é hoje considerada uma doença inflamatória crónica, que, em determinada altura e devido à ruptura da placa, se transforma numa síndrome

\section{isquémica aguda.}

A identificação nas placas ateroscleróticas de linfócitos T e de macrófagos, fez suspeitar do envolvimento dos mecanismos imunológicos na sua etiopatogenia. ${ }^{1}$ Sabe-se agora que as células T e B, os monócitos, as células den- 
dríticas (CD) e as Natural Killer (NK) são fundamentais no desenvolvimento e na progressão da aterosclerose..$^{2-3}$

Um facto que, aparentemente, é indispensável ao desencadear das lesões de aterosclerose, é a penetração e retenção na parede da artéria das lipoproteínas, nomeadamente das LDL. Na parede arterial, as LDL são sujeitas à acção de diversas espécies altamente reactivas, com capacidade oxidativa, sintetizadas pelas células endoteliais, musculares lisas e macrófagos, ${ }^{4-5}$ tornando-as mais imunogénicas, tendo sido já identificadas respostas imunológicas específicas das OxLDL. ${ }^{6}$ Uma das acções fundamentais destas OxLDL é a estimulação da produção de interleucinas, quimiocinas, moléculas de adesão e metaloproteinases pelas células endoteliais, musculares lisas e pelos macrófagos, atraindo as células mononucleares circulantes, induzindo a sua adesão ao endotélio e a sua migração para o espaço sub-endotelial aonde vão desencadear e perpetuar os mecanismos inflamatórios que culminam na formação da placa e posterior ruptura. ${ }^{7-8}$

A maioria dos estudos tem-se debruçado sobre o papel dos macrófagos nos fenómenos inflamatórios e imunológicos subjacentes à fisiopatologia da aterosclerose.

Diversos trabalhos realizados no animal e no homem têm chamado igualmente a atenção para a presença dos linfócitos $T$ e B nas lesões de aterosclerose, realçando o papel destas células na sua fisiopatologia. ${ }^{9}$ Estudos realizados em ratos deficientes em Apo $E\left(A p o E^{-/ /}\right)$e noutros deficientes nos receptores LDL $\left(\mathrm{LDLR}^{-/}\right)$demonstraram que os linfócitos CD4 Th1, produtores de interferão gama e de interleucina 12, terão uma acção predominantemente indutora da aterosclerose, enquanto as células CD4 Th2, produtoras da interleucina 10, terão uma acção essencialmente protectora. ${ }^{10-11}$

Relativamente às células CD4 gostaríamos de nos referir a um subtipo simultaneamente positivo para o CD4 e o CD25, produtor das citocinas anti-inflamatórias IL10 e TGF- $\beta$, conhecidas por CD4+CD25+. Estas células, também nomeadas células $\mathrm{T}$ reguladoras, apresentam propriedades supressoras sobre os linfócitos CD4, possuindo aparentemente propriedades anti-ateroscleróticas. ${ }^{12,13} \mathrm{Em}$ ratos $A$ po $\mathrm{E}^{-1-}$ a quem se administrou células CD4+CD25+, verificou-se uma significativa redução das lesões de aterosclerose. ${ }^{14}$ Indivíduos com síndromes coronárias agudas apresentaram uma redução das percentagens das células CD4+CD25+, assim como uma redução da sua função. ${ }^{15}$

Relativamente aos linfócitos $B$ é mais escassa a literatura sobre a sua potencial acção na doença vascular aterosclerótica. Estas células produzem anticorpos anti-OxLDL e anti-heat shock protein (HSP), que se admite estarem envolvidos na fisiopatologia da aterosclerose. ${ }^{16},{ }^{17}$ Estes anticorpos têm sido detectados em modelos animais de aterosclerose e em indivíduos com doença coronária. ${ }^{18}$ Alguns estudos são compatíveis com um papel protector destes anticorpos relativamente à doença aterosclerótica. A imunização de coelhos e ratos com OxLDL induziu um aumento na produção de anticorpos anti-OxLDL e uma redução das lesões de aterosclerose. ${ }^{28,29} \mathrm{Em}$ ratos simultaneamente ne- gativos para o receptor das LDL e com menos de $1 \%$ de células $B$, alimentados com uma dieta ocidental, verificou-se uma acentuada redução dos títulos de anti-OxLDL e um aumento significativo das lesões de aterosclerose aórtica. ${ }^{30}$

\section{OBJECTIVOS}

Procurámos comparar as populações celulares periféricas existentes em doentes com dislipidemia e doença vascular sub-clínica, avaliada através da determinação da espessura da média-íntima da parede carotídea por eco-doppler e em indivíduos normolipidémicos. No presente artigo debruçámo-nos particularmente sobre os resultados obtidos relativamente às células positivas para o CD4, CD8 e CD19.

\section{MATERIAL E MÉTODOS \\ Caracterização das populações estudadas}

Grupo controlo (C), constituído por 12 indivíduos saudáveis de ambos os sexos (seis homens e seis mulheres), normolipidémicos, com idades compreendidas entre os 30 e os 70 anos (idade média - 51,33 A).

Grupo de dislipidémicos (D), constituído por 38 indivíduos recrutados da nossa Consulta de Dislipidemias, comparáveis em termos de sexo (22 homens e 16 mulheres) e idade (idade média - 55,76) aos do grupo $C$, mas apresentando valores elevados de colesterol total $(>200 \mathrm{mg} / \mathrm{dl})$ ou CLDL (> 130mg/ dl) e / ou triglicerídeos (> 200mg/ dl) e com lesões sub-clínicas de aterosclerose carotídea. Estes doentes não estavam sob terapêutica hipolipemiante pelo menos há um mês, tendo sido mantida, por motivos óbvios, qualquer terapêutica anti-hipertensora, anti-agregante ou qualquer outra considerada indispensável.

Todos os participantes deram o seu consentimento informado, tendo sido o presente estudo aprovado pela Comissão de Ética dos Hospitais da Universidade de Coimbra.

Todos os indivíduos foram submetidos a um exame físico que incluiu a determinação do peso, da altura, do índice de massa corporal, da relação perímetro abdominal / perímetro da anca, da pressão arterial, tendo sido igualmente pesquisadas as manifestações de aterosclerose (sopros vasculares, pulsos periféricos) e as de dislipidemia (arco corneano, xantelasmas / xantomas, hepatoesplenomegalia).

Avaliaram-se as histórias familiares, os hábitos dietéticos, tabágicos e alcoólicos, o uso de fármacos e outras características do estilo de vida.

Analiticamente, dosearam-se a ficha lipídica (o colesterol total, o colesterol HDL, o colesterol LDL e os triglicerídeos), a Apo A1, a Apo B100, a Apo(a) e a genotipagem da Apo $E$, a glucose, o ácido úrico, a CK, a fosfatase alcalina, as transaminases, a gama-GT, as bilirrubinas, o azoto ureico, a creatinina, as hormonas tiroideias e a urina tipo II. Determinaram-se, igualmente, os níveis séricos da ceruloplasmina e da transferrina. Todas as determinações analíticas foram realizadas depois de um jejum de 12 a $14 \mathrm{~h}$.

Realizou-se um electrocardiograma e uma avaliação de eventuais lesões de aterosclerose, com determinação da 
espessura da média-íntima da artéria carotídea, através do estudo com ecodoppler.

Determinou-se igualmente o Índice Perna-Braço (IPB) e quando justificado, realizou-se um doppler arterial dos membros inferiores.

\section{Determinação dos marcadores de membrana}

Os anticorpos monoclonais usados foram:

- CD8 FITC, CD19 FITC, CD3 PE, CD56 PE e CD4 PECy5 -Lymphogram "Cytognos"

- CCR5 PE - RD Systems

- CD8 PE, CD3 Cy5, CD4Cy5 - Dako

- CD95 FITC, CD26 PE, CD62L, HLA DR FITC - Immunotech

- CD4 FITC, CD25 FITC, CD69 PE - CLB

- CD95L PE - Caltag

A aquisição das amostras foi efectuada no citometro de fluxo ('FacsCalibur'-Becton Dickinson) a três fluorescências:

- FL1 - FITC (fluoresceína)

- FL2 - PE (ficoeritrina)

- FL3 - PE Cy5 (ficoeritrina-cianina 5)

Os dados adquiridos foram guardados e analisados posteriormente usando o programa de análise "Paint-agate", que permite identificar as várias populações celulares.

\section{Análise Estatística}

A análise estatística foi realizada com o cálculo de intervalos de confiança a 95\%, e a análise de variância (ANOVA) para comparação de médias, seja entre grupos seja no mesmo grupo em momentos sucessivos. O nível de significância adoptado foi o de $95 \%(p<0,05)$.

Foi utilizado o software estatístico SPSS v.15.

Os resultados estão expressos como números absolutos, relativos e médias (e desvio padrão).

\section{RESULTADOS}

Relativamente aos valores da ficha lipídica a população D apresentou, como seria de esperar, diferenças significativas na concentração do colesterol total $(307,74$ versus $189,67 \mathrm{mg} / \mathrm{dl} ; p=0,000)$ e do colesterol LDL $(172,18$ versus $112,45 \mathrm{mg} / \mathrm{dl} ; p=0,000)$. Os triglicerídeos estavam também mais elevados nos dislipidémicos $(285,47$ versus $142,42 \mathrm{mg} / \mathrm{dl} ; p=0,104)$, embora sem atingir significado estatístico. Relativamente aos valores do colesterol HDL $(49,79$ versus $48,33 \mathrm{mg} / \mathrm{dl} ; p=0,720)$, foram sobreponíveis nas duas populações. A relação CT / CHDL, de grande significado prognóstico e idealmente inferior a cinco, mostrou-se significativamente diferente nas duas populações, com os dislipidémicos apresentando valores que os colocam num risco cardiovascular superior aos controlos $(6,55$ versus 4,05; $p=0,001$ ).

$\mathrm{Na}$ tabela 1 são apresentados os resultados, em percentagem, da determinação das populações celulares periféricas.

Se bem que na sua grande maioria não se tenham ob- servado diferenças significativas nas populações celulares estudadas, entre os indivíduos $\mathrm{D}$ e $\mathrm{C}$, os resultados obtidos justificam alguma reflexão. Tal como dissemos, iremos comentar neste nosso artigo apenas os resultados relativos às células CD4+, CD8+ e CD19+.

As células CD4+ apresentaram-se em percentagem significativamente superior nos controlos, relativamente aos dislipidémicos $(46,233$ versus 40,300; $p=0,026)$.

As percentagens das células CD8+ não mostraram diferenças significativas nos dois grupos de indivíduos $(p=0,783)$.

As células CD19+, tal como as anteriores, encontravam-se em percentagens semelhantes nos dois grupos de participantes $(p=0,945)$

As células positivas para o CD25, que serão referidas a propósito das células reguladoras CD4+CD25+, não determinadas neste nosso estudo, apresentaram-se sobreponíveis nas duas populações $(p=0,108)$, embora tendencialmente mais reduzidas nos dislipidémicos (11,076 versus 12,700).

\section{DISCUSSÃO}

Sabemos que as determinações das populações celulares séricas, poderão não traduzir o que acontece a nível da parede vascular e da lesão de aterosclerose. Por exemplo, as células CD4+ reactivas à HSP humana, foram detectadas na placa de aterosclerose mas não no sangue periféri$\mathrm{co.}^{31} \mathrm{O}$ ideal seria termos amostras tecidulares, mas essa metodologia estava para além das possibilidades do nosso trabalho.

O antigénio CD4 encontra-se nas células T e comporta-se como co-receptor das moléculas da classe II do complexo de histocompatibilidade major (MHC classe II) para activação destas células. É um marcador da diferenciação tímica. Existe também em alguns timócitos, monócitos/macrófagos e granulócitos.

As células CD4+ apresentaram percentagens significativamente inferiores no grupo $D$. A relação entre as células CD4+, os factores de risco e a doença vascular clínica e/ ou sub-clínica tem sido alvo de diversos estudos. Num deles, realizado em homens japoneses com síndrome metabólica, as células CD4+ e CD3+ e as células de memória (CD4+CD45RO+) correlacionaram-se positivamente com o IMC, com as concentrações plasmáticas da glicose e dos triglicerídeos e inversamente com a concentração do CHDL. ${ }^{32}$ Num outro estudo verificou-se, mais uma vez, uma relação positiva entre as células CD4+, CD3+, CD8+ e CD8+CD28+ com a diabetes. ${ }^{19}$ Ainda noutro trabalho, a relação $\mathrm{CD} 4+\mathrm{Th}_{1} / \mathrm{CD} 4+\mathrm{CD} 25+$ esteve positivamente associada com a ocorrência de síndromes coronárias agudas. ${ }^{34}$ Uma correlação positiva foi também encontrada entre os linfócitos CD4+CD28- e o risco de AVC isquémico. ${ }^{20} \mathrm{Re}-$ sultados diferentes foram obtidos num estudo realizado em transplantados renais, em que se verificou uma relação inversa entre os níveis de CD4+ e a ocorrência de eventos cardiovasculares. A uma concentração de CD4+ no maior quartil, correspondeu um risco dez vezes menor ao do associado a uma concentração no menor quartil. ${ }^{21} \mathrm{Um}$ estudo 


\begin{tabular}{|c|c|c|c|c|c|c|c|c|}
\hline \multirow{3}{*}{ CD3 } & \multirow[b]{2}{*}{$\mathrm{D}$} & \multirow{2}{*}{$\frac{\mathbf{N}}{37}$} & \multirow{2}{*}{$\begin{array}{r}\text { Média } \\
73,508\end{array}$} & \multirow{2}{*}{$\begin{array}{r}\begin{array}{r}\text { Desvio } \\
\text { Padrão }\end{array} \\
1,2361\end{array}$} & \multirow{2}{*}{$\begin{array}{r}\begin{array}{c}\text { Erro } \\
\text { Padrão }\end{array} \\
1,2361\end{array}$} & \multicolumn{2}{|c|}{$\begin{array}{c}\text { Interv Confiança } \\
95 \%\end{array}$} & \multirow{2}{*}{$\begin{array}{l}\boldsymbol{P} \\
0,240\end{array}$} \\
\hline & & & & & & 71,001 & 76,015 & \\
\hline & C & 12 & 6,3816 & 1,8422 & 1,8422 & 72,329 & 80,438 & \\
\hline \multirow{2}{*}{ CD4 } & $\mathrm{D}$ & 37 & 40,300 & 7,6530 & 1,2582 & 37,748 & 42,852 & \multirow{2}{*}{0,026} \\
\hline & C & 12 & 46,233 & 8,1492 & 2,3525 & 41,056 & 51,411 & \\
\hline \multirow{2}{*}{ CD8 } & $\mathrm{D}$ & 37 & 23,900 & 6,2737 & 1,0314 & 21,808 & 25,992 & 0,783 \\
\hline & $\mathrm{C}$ & 12 & 24,500 & 7,2770 & 2,1007 & 19,876 & 29,124 & \\
\hline \multirow{2}{*}{ CD19 } & $D$ & 37 & 12,62 & 4,177 & 0,687 & 11,23 & 14,01 & 0,945 \\
\hline & C & 12 & 12,72 & 3,879 & 1,120 & 10,25 & 15,18 & \\
\hline \multirow{2}{*}{ CD56 } & $\mathrm{D}$ & 37 & 13,589 & 6,6275 & 1,0896 & 11,379 & 15,799 & 0,196 \\
\hline & C & 12 & 10,842 & 5,1078 & 1,4745 & 7,596 & 14,087 & \\
\hline \multirow{2}{*}{ CD56CD8 } & $D$ & 37 & 6,035 & 3,2411 & 0,5328 & 4,955 & 7,116 & 0,244 \\
\hline & C & 12 & 4,833 & 2,4103 & 0,6958 & 3,302 & 6,365 & \\
\hline \multirow{2}{*}{ DN } & $D$ & 37 & 3,089 & 2,1216 & 0,3488 & 2,382 & 3,797 & 0,275 \\
\hline & C & 12 & 3,883 & 2,2910 & 0,6614 & 2,428 & 5,339 & \\
\hline & $D$ & 37 & 11,076 & 3,0142 & 0,4955 & 10,071 & 12,081 & 0,108 \\
\hline CD2b & C & 12 & 12,700 & 2,8832 & 0,8323 & 10.868 & 14,532 & \\
\hline & $\mathrm{D}$ & 37 & 39,41 & 11,317 & 1,861 & 35,64 & 43,18 & 0,050 \\
\hline CD26 & C & 12 & 46,99 & 11,392 & 3,289 & 39,75 & 54,23 & \\
\hline & $\mathrm{D}$ & 37 & 8,408 & 2,0973 & 0,3448 & 7,709 & 9,107 & 0,016 \\
\hline CD25CD3 & C & 12 & 10,200 & 2,3460 & 0,6772 & 8,709 & 11,691 & \\
\hline & $D$ & 37 & 36,922 & 11,2472 & 1,8490 & 33,172 & 40,672 & 0,038 \\
\hline CD26CD3 & C & 12 & 44,908 & 11,4119 & 3,2943 & 37,658 & 52,159 & \\
\hline & $\mathrm{D}$ & 37 & 5,157 & 1,7321 & 0,2848 & 4,579 & 5,734 & 0,000 \\
\hline CD25CD26CD3 & $\mathrm{C}$ & 12 & 7,642 & 1,8676 & 0,5391 & 6,455 & 8,828 & \\
\hline & $\mathrm{D}$ & 35 & 2,234 & 1,2943 & 0.2188 & 1,790 & 2,679 & 0,199 \\
\hline CCR5 & C & 12 & 2,942 & 2,3666 & 0,6832 & 1,438 & 4,445 & \\
\hline & $D$ & 35 & 1,603 & 1,0537 & 0,1781 & 1,241 & 1,965 & 0,279 \\
\hline CCR5CD3 & C & 12 & 2,017 & 1,3381 & 0,3863 & 1,166 & 2,867 & \\
\hline & $D$ & 35 & 1,069 & 0,7688 & 0,1299 & 0,804 & 1,333 & 0,867 \\
\hline CCR5CD4 & C & 12 & 1,025 & 0,7944 & 0,2293 & 0,520 & 1,530 & \\
\hline & $\mathrm{D}$ & 37 & 26,489 & 7,7643 & 1,2764 & 23,900 & 29,078 & 0,014 \\
\hline HLARD & C & 12 & 20,308 & 5,2219 & 1,5074 & 16,991 & 23,626 & \\
\hline НI $\triangle \mathrm{RPCD4}$ & $\mathrm{D}$ & 37 & 7,722 & 3,8419 & 0,6316 & 6,441 & 9,003 & 0,015 \\
\hline HLADRCD4 & C & 12 & 4,508 & 3,7833 & 1,0922 & 2,105 & 6,912 & \\
\hline & $\mathrm{D}$ & 37 & 4,486 & 1,9518 & 0,3209 & 3,836 & 5,137 & 0,001 \\
\hline HLADRCD8h & C & 12 & 2,325 & 1,6526 & 0,4771 & 1,275 & 3,375 & \\
\hline & $\mathrm{D}$ & 37 & 1,557 & 0,9648 & 0,1586 & 1,235 & 1,878 & 0,027 \\
\hline HLADRCD8low & $\mathrm{C}$ & 12 & 0,843 & 0,8695 & 0,2510 & 0,291 & 1,396 & \\
\hline Нا & $\mathrm{D}$ & 37 & 6,068 & 2,5047 & 0,4118 & 5,232 & 6,903 & 0,001 \\
\hline HLADRCD8 & C & 12 & 3,167 & 2,3689 & 0,6838 & 1,662 & 4,672 & \\
\hline & $\mathrm{D}$ & 37 & 21,076 & 11,2630 & 1,8516 & 17,320 & 24,831 & 0,487 \\
\hline CD95 & C & 12 & 18,650 & 7,0150 & 2,0251 & 14,193 & 23,107 & \\
\hline & $\mathrm{D}$ & 37 & 0,470 & 0,4169 & 0,0685 & 0,331 & 0,609 & 0,064 \\
\hline CD95CD95L & C & 12 & 0,235 & 0,1699 & 0,0490 & 0,127 & 0,343 & \\
\hline & $\mathrm{D}$ & 37 & 17,511 & 10,3625 & 1,7036 & 14,056 & 20,966 & 0,966 \\
\hline CD3CD95 & C & 12 & 12,375 & 6,8094 & 1,9657 & 13,049 & 21,701 & \\
\hline & $\mathrm{D}$ & 37 & 1,219 & 1,2778 & 0,2101 & 0,793 & 1,645 & 0,400 \\
\hline CD3CD95L & $\mathrm{C}$ & 12 & 0,892 & 0,6302 & 0,1819 & 0,491 & 1,292 & \\
\hline & $D$ & 37 & 60,046 & 14,3597 & 2,3607 & 55,258 & 64,834 & 0,087 \\
\hline CD62L & C & 12 & 67,933 & 10,5455 & 3,0442 & 61,233 & 74,634 & \\
\hline Cח3CD621 & $D$ & 37 & 43,792 & 12,8940 & 2,1198 & 39,493 & 48,091 & 0,066 \\
\hline CD3CD62L & C & 12 & 51,700 & 11,8714 & 3,4270 & 44,157 & 59,243 & \\
\hline Cח69 & $D$ & 37 & 11,705 & 7,0168 & 1,1536 & 9,366 & 14,045 & 0,024 \\
\hline CD69 & C & 12 & 16,750 & 4,5900 & 1,3250 & 13,834 & 19,666 & \\
\hline CD69c03 & $\mathrm{D}$ & 37 & 5,468 & 4,2780 & 0,7033 & 4,041 & 6,894 & 0,003 \\
\hline CD69CD3 & C & 12 & 9,758 & 3,7922 & 1,0947 & 7,349 & 12,168 & \\
\hline Cח69cR4 & $\mathrm{D}$ & 37 & 3,511 & 2,8682 & 0,4715 & 2,555 & 4,467 & 0,008 \\
\hline CD69CD4 & C & 12 & 6,125 & 2,6840 & 0,7748 & 4,420 & 7,830 & \\
\hline
\end{tabular}


em doentes HIV+ em que, tal como no nosso, se avaliou a doença carotídea por doppler, uma maior concentração de células CD4+ associou-se a uma menor progressão da doença carotídea $(p=0,01) .{ }^{22}$ Por outro lado, em indivíduos saudáveis, entre os 60 e os 70 anos, as células T de memória CD4+CD45RO+e as B activadas CD19+CD80+ correlacionaram-se positiva e significativamente, com a espessura da média-íntima carotídea. ${ }^{23}$

O CD8 encontra-se nas células T, sendo um co-receptor para a activação destas células que reconhecem os antigénios ligados às moléculas da classe I do MHC. Está também presente em alguns timócitos e em algumas células dendríticas.

As células CD8+ comportam-se como células citotóxicas, eliminando as células infectadas com vírus, as células tumorais e as células de enxerto e não apresentaram diferenças percentuais significativas entre os indivíduos dos grupos D e C.

São em menor quantidade os trabalhos que avaliaram uma possível relação entre as células CD8+ do sangue periférico e a doença vascular. Um deles foi já referido. ${ }^{33}$ Noutro, realizado em doentes com apneia do sono, em que a doença vascular tem uma prevalência superior ao da população geral, verificou-se que os linfócitos CD8+ periféricos apresentavam um grau de citotoxicidade, traduzida pela presença de CD56 e CD16, significativamente superior aos dos controlos. ${ }^{24}$ Resultados semelhantes foram obtidos num outro estudo. ${ }^{25}$

A percentagem significativamente maior das células CD4+ $(0,026)$ nos controlos e a semelhança das CD8+ nos dois grupos de indivíduos $(p=0,783)$, poderá fazer antever um maior peso da imunidade humoral $\left(\mathrm{CD} 4 \mathrm{TH}_{2}\right)$ e lou da hipersensibilidade celular $\left(\mathrm{CD} 4 \mathrm{TH}_{1}\right)$ nos normolipidémicos, assim como um predomínio da imunidade celular (CD8+) nos dislipidémicos do nosso estudo.

Com a ressalva de não ter sido estudada a artéria carotídea nos nossos controlos, admitimos como provável que os indivíduos dislipidémicos apresentem lesões mais avançadas e/ou mais instáveis. Sendo assim, somos tentados a concordar com alguns autores que referem ser a imunidade celular a principal responsável pela doença aterosclerótica, atribuindo à imunidade humoral, particularmente aos anticorpos anti-OxLDL, um papel protector. ${ }^{26}$ Este conceito está longe de ser consensual, com alguns trabalhos a demonstrarem resultados contraditórios. ${ }^{27}$

Gostaríamos de chamar a atenção para um facto que julgamos importante. Não tendo sido analisada a percentagem das células duplamente positivas para o CD4 e o CD25, células CD4+CD25+, com funções reguladoras, verificou-se que a percentagem dos marcadores CD4 e CD25 estava reduzida nos nossos dislipidémicos (os segundos sem significado). Dada a importância atribuída às células CD4+CD25+ na fisiopatologia da aterosclerose, inibindo o seu desenvolvimento, ${ }^{23,28,29}$ poderíamos especular que a simultaneidade de valores mais baixos de CD4 e de CD25 nos participantes do nosso grupo $D$, poderiam traduzir uma reduzida percentagem das células $T$ reguladoras CD4+CD25+ nos nossos dislipidémicos!

Relativamente às células $B$, identificadas pelo CD19, parece exercerem um papel essencialmente protector relativamente ao desenvolvimento da aterosclerose e encontravam-se em percentagens semelhantes nos dois grupos de indivíduos. Esta acção protectora ficou demonstrada em diversos estudos animais em que se verificou que a sua concentração na parede do vaso foi sofrendo uma progressiva diminuição à medida que aumentou a área ocupada pelas placas ${ }^{3}$ e que a deficiência congénita dos linfócitos CD19+ se associou a um maior desenvolvimento das lesões de aterosclerose. ${ }^{30}$

Resumindo, os resultados obtidos relativamente às percentagens das populações celulares periféricas, permitem-nos algumas reflexões:

Relativamente à menor concentração das células CD4+ na nossa população de dislipidémicos, aparentemente inesperada devido ao relacionamento existente entre este tipo celular, os factores de risco e a aterosclerose, seria necessário caracterizar melhor o tipo de células CD4+. Com efeito e como já referimos, a simultaneidade de menores percentagens de CD4 e de CD25 (estas sem significado) na nossa população $\mathrm{D}$, permite-nos especular que poderão ser essencialmente as células CD4+CD25+, reguladoras e com funções supressoras da aterosclerose, que poderão estar reduzidas nestes indivíduos. ${ }^{23}$ Também o fenótipo apresentado pelas células CD4+, essencialmente Th1, com produção de Interferão gama, ou Th2, com produção de citocinas anti-inflamatórias, como a IL10, poderá explicar a relação entre estas células, os factores de risco e o desenvolvimento da aterosclerose. ${ }^{17-20} \mathrm{~A}$ semelhança nas concentrações das células CD8+ nos dois grupos, acompanhada de uma significativa redução das CD4+ nos dislipidémicos, faz supor um predomínio da imunidade celular nestes últimos indivíduos.

\section{CONCLUSÃO}

O nosso estudo apresenta algumas limitações. Desde logo a pequena dimensão das populações, particularmente do grupo dos controlos. Também o facto de alguns dos indivíduos dislipidémicos terem estado medicados com hipolipemiantes até um mês antes da determinação das populações celulares, poderá ter interferido com as percentagens destas. Cerca de dezasseis dos dislipidémicos estavam medicados com ácido acetilsalicílico em doses antiagregantes. Apesar das baixas doses utilizadas, entre 100 a $150 \mathrm{mg} / \mathrm{dia}$, poderem excluir efeitos antinflamatórios significativos, alguns estudos conseguiram demonstrar uma redução significativa de moléculas como a PCR, obtida com ácido acetilsalicílico nas doses de 75 a $300 \mathrm{mg} /$ dia. $^{31,32}$ 


\section{CONFLITOS DE INTERESSE}

Os autores declaram a inexistência de conflitos de interesse na realização do presente trabalho.

\section{REFERÊNCIAS}

1. Hansson GK. Inflammation, atherosclerosis, and coronary artery disease. N Engl J Med. 2005;352:1685-95.

2. Zhou X, Nicoletti A, Elhage R, Hansson GK. Transfer of CD4(+) T cells aggravates atherosclerosis in immunodeficient apolipoprotein $\mathrm{E}$ knockout mice. Circulation. 2000;102:2919-22.

3. Major AS, Fazio S, Linton MF. B-lymphocyte deficiency increases atherosclerosis in LDL receptor-null mice. Arterioscler Thromb Vasc Biol. 2002;22:1892-8

4. Aqel NM, Ball RY, Waldmann H, Mitchinson MJ. Monocytic origin of foam cells in human atherosclerotic plaques. Atherosclerosis. 1984; 53:265-71.

5. Yilmaz A, Lochno M, Traeg F, Cicha I, Reiss C, Stumpf C, et al. Emergence of dendritic cells in rupture-prone regions of vulnerable carotid plaques. Atherosclerosis. 2004;176:101-10.

6. Whitman SC, Rateri DL, Szilvassy SJ, Yokoyama W, Daugherty A. Depletion of natural killer cell function decreases atherosclerosis in lowdensity lipoprotein receptor null mice. Arterioscler Thromb Vasc Biol. 2004;24:1049-54.

7. Tupin E, Nicoletti A, Elhage R, Rudling M, Ljunggren HG, Hansson GK, Berne GP. CD1d-dependent activation of NKT cells aggravates atherosclerosis. J Exp Med. 2004;199:417-22.

8. Witztum JL, Steinberg D. Role of oxidized low density lipoprotein in atherogenesis. J Clin Invest. 1991;88:1785-92.

9. Parthasarathy $S$. Modified lipoproteins in the pathogenesis of atherosclerosis. Austin: RG Landes Co.; 1994.

10. Parthasarathy S. Mechanism(s) of cell-mediated oxidation of low density lipoprotein. In: Nohl H, Esterbauer H, Rice Evans C, editors. Free Radicals in the Environment, Medicine and Toxicology. London: Richelieu Press; 1994. p.163-79.

11. Witztum JL. The oxidation hypothesis of atherosclerosis. Lancet. 1994;344:793-5

12. Paulsson G, Zhou X, Törnquist E, Hansson GK. Oligoclonal T cell expansions in atherosclerotic lesions of apolipoprotein E-deficient mice. Arterioscler Thromb Vasc Biol. 2000;20:10-7.

13. Sultana C, Shen Y, Rattan V, Kalva VK. Lipoxygenase metabolitos induced expresión of adhesión and transendothelial migration of monocyte-like HL-60 cells is linked to protein kinase C activation. J Cell Physiol. 1996;167:477-87.

14. Khan B, Parthasarathy S, Alexander R, Medford R. Modified low density lipoprotein and its constituents augment cytokine-activated vascular cell adhesion molecule-1 gene expression in human vascular endothelial cells. J Clin Invest. 1995;95:1262-70.

15. Xu X, Meisel S, Ong J, Kaul S. Oxidized low-density lipoprotein regulates matrix metalloproteinase- 9 and its tissue inhibitor in human monocyte-derived macrophages. Circulation. 1999;99:993-8.

16. de Boer OJ, Becker AE, van der Wal AC. T lymphocytes in atherogenesis-functional aspects and antigenic repertoire. Cardiovasc Res. 2003;60:78-86.

17. Daugherty A, Pure E, Delfel-Butteiger D, Chen S. The effects of total lymphocyte deficiency on the extent of atherosclerosis in apolipoprotein E-/- mice. J Clin Invest. 1997;100:1575-80.

18. Reardon CA, Blachowicz L, White T, Cabana V. Effect of immune deficiency on lipoproteins and atherosclerosis in male apolipoprotein Edeficient mice. Arterioscler Thromb Vasc Biol. 2001;21:1011-6.

19. Lee TS, Yen HC, Pan CC, Chau LY. The role of interleukin 12 in the development of atherosclerosis in apoE-deficient mice. Arterioscler Thromb Vasc Biol. 1999;19:734-42.

20. Pinderski Oslund LJ, Hedrick CC, Olvera T, Hagenbaugh A. Interleukin-10 blocks atherosclerotic events in vitro and in vivo. Arterioscler Thromb Vasc Biol. 1999;19:2847-53.

21. Robertson AK, Hansson GK. T Cells in atherogenesis: for better or for worse? Arterioscler Thromb Vasc Biol. 2006;26:2421-32.

22. Smith DA, Irving SD, Sheldon J, Cole D, Kaski JC. Serum levels of the antiinflammatory cytokine interleukin-10 are decreased in patients with unstable angina. Circulation. 2001;104:746-9.

23. Mor A, Planer D, Luboshits G, Afek A. Role of naturally ocurring CD4+CD25+ regulatory Tcells in experimental atherosclerosis. Arterioscler Thromb Vasc Biol. 2007;27:893.

24. Mor A, Luboshits G, Planer D, Keren G. Altered status of CD4+CD25+

\section{FONTES DE FINANCIAMENTO}

Não existiram fontes externas de financiamento para a realização deste artigo.

regulatory T cells, in patients with acute coronary syndromes. Eur Heart J. 2006;27:2530-7.

25. Shaw PX, Horkko S, Chang MK, Curtiss LK. Natural antibodies with the T15 idiotype may act in atherosclerosis, apoptotic clearance, and protective immunity. J Clin Invest. 2000;105:1731-40.

26. Wick G. Atherosclerosis: an autoimmune disease due to an immune reaction against heat-shock protein 60. Herz. 2000;25:87-90.

27. Tsimikas S, Palinski W, Witztum JL. Circulating autoantibodies to oxidized LDL correlate with arterial accumulation and depletion of oxidized LDL in LDL receptor-deficient mice. Arterioscler Thromb Vasc Biol. 2001;21:95-100.

28. Freigang S, Horkko S, Miller E, Witztum JL. Immunization of LDL receptor-deficient mice with homologous malondialdehyde-modified and native LDL reduces progression of atherosclerosis by mechanisms other than induction of high titers of antibodies to oxidative neoepitopes. Arterioscler Thromb Vasc Biol. 1998;18:1972-82.

29. Palinski W, Miller E, Witztum JL. Immunization of low density lipoprotein (LDL) receptor-deficient rabbits with homologous malondialdehyde-modified LDL reduces atherogenesis. Proc Natl Acad Sci USA. 1995;92:821-5.

30. Major A, Fazio S, Linton MacRae. B-Lymphocyte deficiency increases atherosclerosis in LDL receptor-null mice. Arterioscler Thromb Vasc Biol. 2002;22:1892

31. Benagiano M, D’Elios M, Amedei A, Azzuri A. Human 60-KDa shock protein is a target autoantigen of $T$ cells derived from atherosclerotic plaques. J Immunol. 2005;174:6509-17.

32. Tanigawa $\mathrm{T}$, Iso $\mathrm{H}$, Yamagishi K, Muraki I. Association of lymphocyte sub-population with clustered features of metabolic syndrome in middleaged Japanese men. Atherosclerosis. 2004;173:295-300.

33. Dworacka M, Winiarska H, Borowska M, Abramczyk M. Pro-atherogenic alterations in T-lymphocyte subpopulations related to acute hyperglycaemia in type 2 diabetic patients. Circ J. 2007;71:962-7.

34. Han SF, Liu P, Zhang W, Bu L. The opposite-direction modulation of CD4+CD25+ Tregs and T helper 1 cells in acute coronary syndromes. Clin Immunol. 2007;124:90-7.

35. Nowik M, Nowacki P, Grabarek J, Drechsler H. Can we talk about CD4+CD28- lymphocytes as a risk factor for ischemic stroke? Eur Neurol. 2007;58:26-33.

36. Ducluoux D, Challier B, Sas P, Tiberghien P. CD4 cell lymphopneia and atherosclerosis in renal transplant recipients. J Am Soc Nephrol. 2003;14:767-72.

37. Coll B, Parra S, Alonso-Villaverde C, Aragonês G, Montero M. The role of immunity and inflammation in the progression of atherosclerosis in patients with HIV infection. Stroke. 2007;38:2477-84.

38. Tanigawa T, Kitamura A, Yamagashi K, Sakurai S. Relationships of differential leucocyte and lymphocyte subpopulations with carotid atherosclerosis in elderly men. J Clin Immunol. 2003;23:469-76.

39. Dyugovskaya L, Lavie P, Hirsh M, Lavie L. Activated CD8+ T-lymphocytes in obstrutive sleep apnea. Eur Respir J. 2005;25:820-8.

40. Dyugovskaya L, Lavie P, Lavie L. Lymphocite activation as a possible measure of atherosclerotic risk in patients with sleep apnea. Ann N Y Acad Sci. 2005;1501:340-50.

41. Wu R, de Faire U, Lemme C, Witztum L. Autoantibodies to oxLDL are decreased in individuals with borderline hipertension. Hypertension.1999;33:53-5.

42. Meraviglia M, Maggi E, Bellomo G, Cursi M. Autoantibodies against oxidatively modified lipoproteins and progression of carotid endarterectomy. Stroke. 2002;33:1139.

43. Nilsson J. Regulating protective immunity in atherosclerosis. Circ Res. 2005;96:395-7.

44. Gotsman I, Gupta R, Lichtman A. The Influence of the regulatory $\mathrm{T}$ lymphocytes on atherosclerosis. Arterioscler Thromb Vasc Biol. 2007;27:2493.

45. Galkina E, Kadl A, Sanders J, Varughese D. Lymphocyte recruitment into the aortic wall before and during development of atherosclerosis is partially L-selectin dependent. J Exp Med. 2006;203:1273-82.

46. Woodward M, Lowe GD, Francis LM, Rumley A, Cobbe SM. A randomized comparison of the effects of aspirin and clopidogrel on thrombotic risk factors and C-reactive protein following myocardial infarction: the 
47. CADET trial. J Thromb Haemost. 2004;2:1934-40.

47. Ikonomidis I, Lekakis J, Vamvakou G, Andreotti F. Cigarette smoking is associated with increased circulating proinflammatory and procoagulant markers in patients with chronic coronary artery disease: effects of aspirin treatment. Am Heart J. 2005;149:832-9. 
José PEREIRA de MOURA, Manuel SANTOS ROSA, Vera ALVES, Anabela MOTA PINTO, Victor RODRIGUES, José Manuel SILVA, J.J. ALVES de MOURA

\section{Populações Celulares Periféricas (CD4+, CD8+ e CD19+) em Indivíduos Dislipidémicos \\ Acta Med Port 2013:26:676-682}

Publicado pela Acta Médica Portuguesa, a Revista Científica da Ordem dos Médicos

Av. Almirante Gago Coutinho, 151

1749-084 Lisboa, Portugal.

Tel: +351218428 215

E-mail: submissao@actamedicaportuguesa.com

www.actamedicaportuguesa.com

ISSN:0870-399X | e-ISSN: 1646-0758



